\title{
Linear stability analysis of an insoluble surfactant monolayer spreading on a thin liquid film
}

\author{
Omar K. Matar and Sandra M. Troian \\ Department of Chemical Engineering, Princeton University, Princeton, New Jersey 08544-5263
}

(Received 17 March 1997; accepted 11 August 1997)

\begin{abstract}
Recent experiments by several groups have uncovered a novel fingering instability in the spreading of surface active material on a thin liquid film. The mechanism responsible for this instability is yet to be determined. In an effort to understand this phenomenon and isolate a possible mechanism, we have investigated the linear stability of a coupled set of equations describing the Marangoni spreading of a surfactant monolayer on a thin liquid support. The unperturbed flows, which exhibit simple linear behavior in the film thickness and surfactant concentration, are self-similar solutions of the first kind for spreading in a rectilinear geometry. The solution of the disturbance equations determines that the rectilinear base flows are linearly stable. An energy analysis reveals why these base flows can successfully heal perturbations of all wavenumbers. The details of this analysis suggest, however, a mechanism by which the spreading can be destabilized. We propose how the inclusion of additional forces acting on the surfactant coated spreading film might give rise to regions of adverse mobility gradients known to produce fingering instabilities in other fluid flows. (C) 1997 American Institute of Physics. [S1070-6631(97)00112-8]
\end{abstract}

\section{INTRODUCTION}

The spreading of solutions containing surface active material plays a very significant role in daily life and industry ranging from detergency and aerosol delivery of medicating drugs to lubrication and ink jet printing. In general, a pure liquid film spreading on a dry solid will advance fairly slowly unless driven by external forces like gravity, centrifugation, or surface shear. A surface active film distributed on a thin liquid support, however, will cause spontaneous and very rapid spreading when the surface material creates regions of lower surface tension than the supporting liquid. Controlling the rate and extent of such spreading requires a thorough understanding of the so-called Marangoni flow. Typical coating solutions often contain surface active agents like hydrocarbon solvents, phospholipids, surfactants or dyes. Not only do these substances significantly lower the surface tension of the supporting liquid but, depending on their local concentration, will create gradients in surface tension along the air-liquid interface.

While conducting experiments on the radial advance of small water droplets containing various surfactants on the surface of glass, Marmur and Lelah ${ }^{1}$ first reported the presence of dendritic-like patterns during the spreading process. They assumed that such unusual patterns were formed during the spreading of a droplet on a dry substrate whenever the bulk surfactant concentration was above the critical micelle concentration (CMC). Marmur and Lelah speculated that a primary film of surfactant spread out onto the dry substrate ahead of the macroscopic drop and that this precursor film adsorbed onto the glass surface in one of three orientational modes. These deposition modes were believed to form hydrophilic and hydrophobic patches on the substrate which ultimately gave rise to the intricate wetting patterns observed. By repeating these experiments in a controlled humidity cell to prevent spurious evaporation from the thinnest parts of the spreading films, Troian et al. ${ }^{2}$ observed much more regular fingering patterns as shown in Fig. 1. Such patterns were obtained by gently depositing a $2 \mu \mathrm{l}$ droplet of $1 \mathrm{mM}$ AOT [sodium bis-(2-ethylhexyl) sulfosuccinate] on the surface of a clean water film ranging in thickness from 1 $\mathrm{mm}$ to fractions of a micron. Many common laboratory detergents were found to produce a similar instability. ${ }^{1-3}$ In each case, the spreading droplet creates a rapidly growing disk with a thickened front that advances with a speed on the order of $\mathrm{cm} / \mathrm{s}$. Far behind this advancing rim, there develops a sharp depression in liquid height near the location of the initial deposition point. Slender fingers appear and grow rapidly into this thinned region undergoing spreading, shielding, and tip-splitting, processes observed in other fluid flow instabilities driven by completely different forces. ${ }^{4}$ During the past few years several other experimental groups have since confirmed the development of this fingering instability during the spreading of insoluble and soluble surfactant films on a thin water support. ${ }^{3,5-7}$

Troian $e t$ al. $^{2}$ detected the instability for surfactant concentrations both above and below the CMC. More importantly, the fingering patterns never appeared on perfectly dry substrates. This last observation coupled with the rapid spreading velocities suggested that the fingering instability derives from the presence of Marangoni effects. In fact, the rapid spreading and fingering were shown to depend on the overall difference in surface tension between the pure and contaminated water surface and the initial thickness of the water support, in agreement with flows driven by Marangoni stresses. Preliminary image analysis studies of the spreading fronts also uncovered that the contours were fractal curves ${ }^{8}$ of fractal dimension close to the values obtained in physical systems governed by Laplacian-type kinetics like diffusion limited aggregation, viscous fingering, or dendritic growth. This last feature led the authors to investigate mathematical similarities to other fluid systems governed by Laplacian fields ${ }^{9}$ and provided the first attempt at a stability analysis for Marangoni driven spreading. 


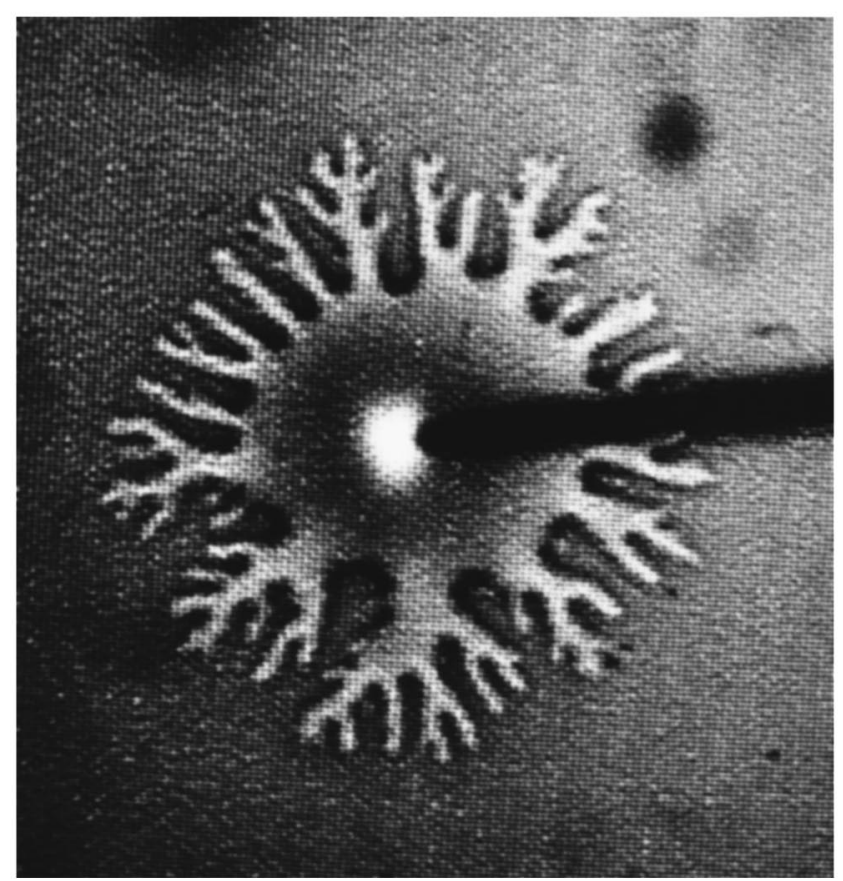

(a)

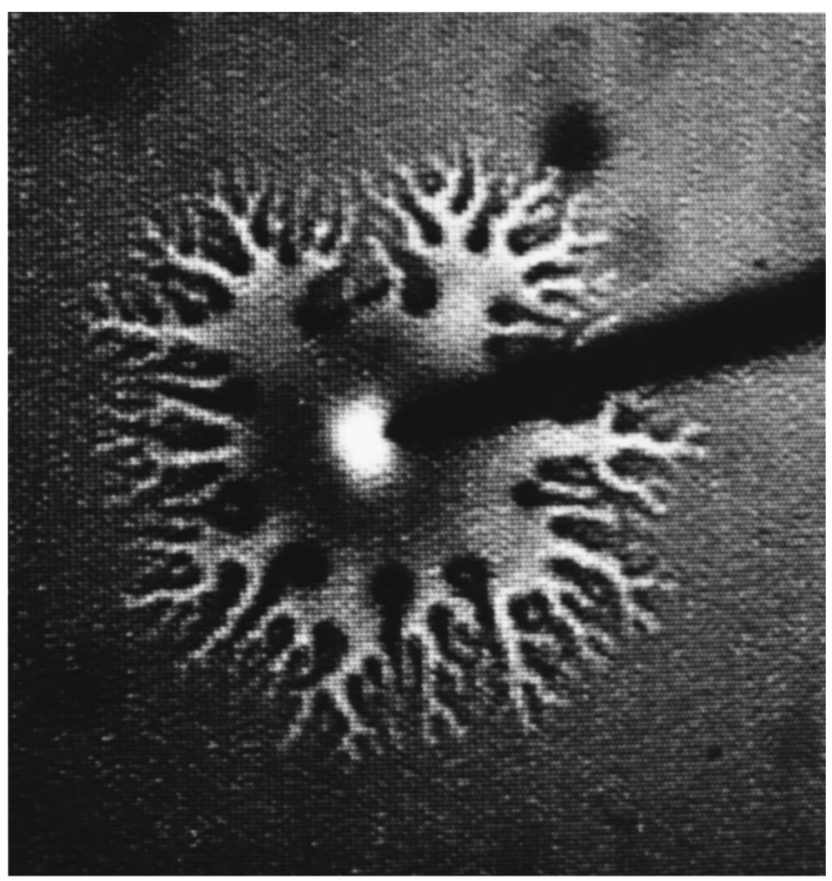

(b)

FIG. 1. The spreading pattern generated by a $2 \mu \mathrm{l}$ drop of $1 \mathrm{mM}$ aqueous AOT solution deposited on a thin water film. ${ }^{2}$ Initial water film thickness: (a) $H \approx 1 \mu \mathrm{m}$ and (b) $H \approx 0.1 \mu \mathrm{m}$.

The original geometry used to model this new Marangoni instability was a hemispherical cap of fluid of variable volume coated with insoluble surfactant spreading on a clean thin liquid film consisting of the same fluid. ${ }^{9}$ In this model, the drop provides a large reservoir of surfactant delivered as a monolayer along the uncontaminated liquid film. A scaling analysis in rectilinear geometry determines that the advancing surfactant coated film spreads in time as $t^{1 / 2}$. This rapidly moving front was shown to control the spreading rate of the hemispherical droplet which also spread as $t^{1 / 2}$. At long times, when Marangoni stresses decay significantly and the spreading is instead controlled by capillary forces, the spreading rate was shown to decrease substantially and approach the dynamics of a capillary driven flow. An asymptotic analysis revealed that Marangoni stresses create a long thin region of length, $L(t)$, ahead of the macroscopic droplet in which a sizeable gradient in surfactant concentration is established. At the leading front where the spreading monolayer joins the motionless uncontaminated film, a sharp front is formed of height almost twice the undisturbed thickness. Capillary terms arising from regions of strong curvature are only significant in two small regions of length $l(t)$ $\ll L(t)$ located near the base of the droplet reservoir and at the steep front. The numerically calculated film thickness profiles, including capillary effects, indicated significant thinning near the point of deposition and a corresponding thickening at the advancing edge. This long Marangoni controlled region appeared to develop a quasi-linear increase in thickness and a corresponding linear decrease in surfactant concentration. For long wavelengths $\lambda$ in the range $l(t) \ll \lambda$ $\ll L(t)$, a simplified linear stability analysis concluded that the flow is unstable to perturbations at the base of the spreading drop. To leading order in the wavenumber, the perturbed concentration field was shown to satisfy Laplace's equation provided the disturbances in the film thickness were assumed negligible in comparison to disturbances in the surfactant concentration. In this work, we revisit Marangoni driven flow and provide a more rigorous and complete formulation of the disturbance analysis.

Within the lubrication approximation, Marangoni driven spreading is described by a coupled set of nonlinear partial differential equations whose solutions provide the spatiotemporal evolution for the film thickness and surfactant concentration. Recent analysis of these equations have determined that there are several self-similar solutions to the unperturbed flow depending on geometry and surfactant feeding rate ${ }^{10,11}$ for purely Marangoni driven spreading. In this paper we focus primarily on Marangoni driven spreading in the simplest geometry allowable, namely the rectilinear spreading of a finite monolayer of insoluble surfactant spreading on the surface of a thin liquid film of higher tension. This geometry affords the fastest spreading rate for a finite quantity of surfactant. We study the linear stability of the rectilinear solution whose front advances as $t^{1 / 3}$ in time. The calculations in the present work differ from these earlier studies in two main ways. First, we ignore capillary forces in order to derive simple self-similar solutions to the base flows. Second, we allow for disturbances in both the film thickness and surfactant concentration. Even within a quasisteady state approximation, the analysis is not straightforward. The coupled disturbance equations contain two regular singular points associated with the linear self-similar solutions. The troublesome singularity at the origin is identified by a Frobenius expansion and removed analytically. A proper mathematical treatment of the associated eigenvalue equations determines that the rectilinear self-similar solutions are linearly stable to perturbations of all wavenumbers.

Inspection of the linearly stable eigenfunctions reveals a 


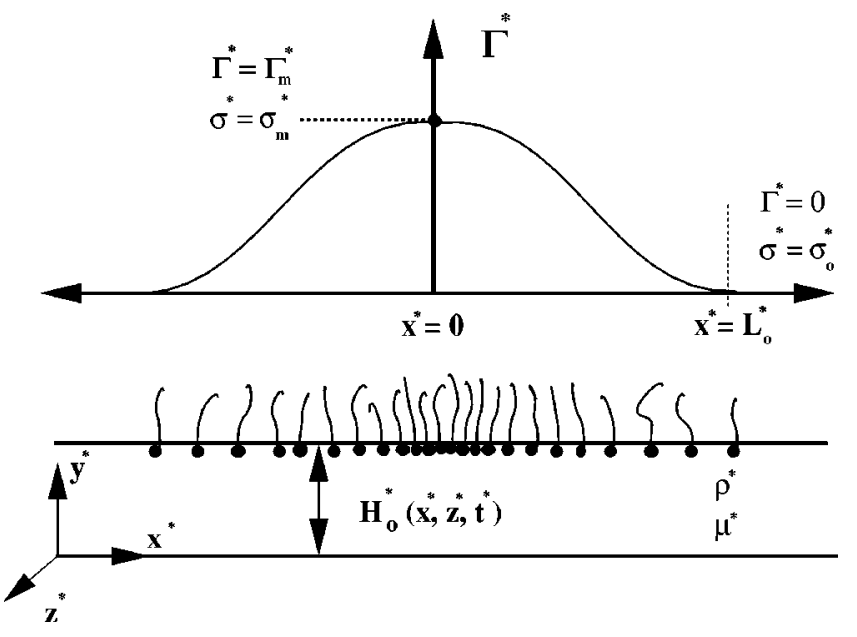

FIG. 2. Schematic diagram of the spreading process.

rich structure in Fourier space whose stability characteristics are better revealed by a complete energy analysis. This analysis demonstrates how the Marangoni contributions created by the disturbance flow in the transverse direction are strong enough to dampen disturbances of all wavenumbers and to restore the system to its original self-similar form. By inspecting the various contributions to the rate of energy production in the system, we suggest what types of eigenfunction solutions could destabilize the flow. In particular, solutions of the flow for which a local decrease in film thickness is accompanied by a local increase in surfactant concentration could overturn the energy balance provided such contributions were sizeable. We also discuss how the addition of capillary and surface diffusion terms into the original equations of motion will modify the spreading profiles to produce regions of adverse mobility near the point of deposition and near the advancing front. Regions of adverse mobility gradients have been invoked in the past to explain the source of instability in viscous fingering problems, for example. Although the physics driving the Marangoni spreading problem is significantly different than the physics driving viscous fingering phenomena ${ }^{12}$ (i.e., Marangoni spreading is described by two coupled flow variables and requires no external driving force) this same general concept may help uncover the source of the fingering instability in Marangoni flows.

\section{FORMULATION OF GENERAL BASE STATE}

\section{A. Base state}

Consider a thin Newtonian liquid film of initial uniform thickness $H_{0}^{*}\left(x^{*}, z^{*}, t^{*}\right)$, viscosity $\mu^{*}$, and density $\rho^{*}$ resting on a flat solid substrate whose surface is located at $y^{*}$ $=0$. The coordinate $x^{*}$ denotes the horizontal coordinate, $y^{*}$ the vertical coordinate and $z^{*}$ the transverse coordinate. The liquid film is partially covered by an insoluble surfactant monolayer whose surface concentration, $\Gamma^{*}\left(x^{*}, z^{*}, t^{*}\right)$, varies smoothly from its maximum value, $\Gamma_{m}^{*}$, at the origin $x^{*}=0$, to a value of zero at $x^{*}=L_{0}^{*}$, as shown in Fig. 2 . The quantities $H_{0}^{*}$ and $L_{0}^{*}$ are used to scale all vertical and horizontal displacements, respectively. Upon deposition, the monolayer will spread rapidly and spontaneously, driven by the initial spreading pressure $\Pi_{0}^{*}=\sigma_{0}^{*}-\sigma_{m}^{*}$, where $\sigma_{0}^{*}$ denotes the surface tension of the clean liquid surface and $\sigma_{m}^{*}$ the surface tension of the contaminated liquid ${ }^{13}$ at the origin of flow $x^{*}=0$. We designate by $\epsilon$ the ratio of the initial film thickness, $H_{0}^{*}$, to the initial extent of surfactant coverage, $L_{0}^{*}$, which small parameter in lubrication theory satisfies $\epsilon$ $\ll 1$. ${ }^{14}$ Initial gradients in surface tension of order $\Pi_{0}^{*} / L_{0}^{*}$ generate shear stresses at the interface of order $\mu^{*} U^{*} / H_{0}^{*}$. The characteristic velocity for the streamwise and transverse directions is, therefore, $U^{*}=\epsilon \Pi_{0}^{*} / \mu^{*}$, while the vertical velocity is scaled by $\epsilon U^{*}$. According to the lubrication approximation, the pressure is scaled by $P^{*}=\mu^{*} U^{*} L_{0}^{*} / H_{0}^{* 2}$. Insertion of the Marangoni velocity, $U^{*}$, into the pressure scaling yields $P^{*}=\Pi_{0}^{*} / H_{0}^{*}$, in which the force per unit length, $\Pi_{0}^{*}$, has effectively been converted into a force per unit area $P^{*}$. The dimensionless surface pressure is defined as $\sigma=\left(\sigma^{*}-\sigma_{m}^{*}\right) / \Pi_{0}^{*}$, which describes the ratio of the driving force at any point on the film surface to the maximum driving force. Introduction of these scalings into the equations of incompressibility and momentum conservation for the liquid support yields the following equations in dimensionless form:

$$
\begin{aligned}
& u_{x}+v_{y}+w_{z}=0, \\
& 0=-p_{x}+u_{y y}+O\left(\epsilon R e, \epsilon^{2}\right), \\
& 0=-p_{y}+B o+O\left(\epsilon^{2}\right), \\
& 0=-p_{z}+w_{y y}+O\left(\epsilon R e, \epsilon^{2}\right),
\end{aligned}
$$

wherein the axial, vertical and transverse velocity fields are represented by $u, v$ and $w$, respectively, and henceforth, subscripts refer to differentiation by $x, y, z$ and $t$ unless otherwise stated. With this choice of scales the modified Bond number is defined as $B o \equiv\left(\rho^{*} g^{*} \epsilon^{2} L_{0}^{* 2}\right) / \Pi_{0}^{*}$ while the modified Reynolds number is given by $R e$ $\equiv\left(\rho^{*} U^{*} H_{0}^{*}\right) / \mu^{*}=\left(\rho^{*} \Pi_{0}^{*} \epsilon^{2} L_{0}^{*}\right) / \mu^{* 2}$. The parameter $B o$ represents the ratio of hydrostatic pressure to the Marangoni stress while $R e$ represents the ratio of inertial forces to viscous forces, in which $U^{*}$ is set by the velocity governing Marangoni convection. Since $B o$ and $R e$ both scale as $\epsilon^{2}$, all such terms are dropped from consideration in this analysis correct to $O(\epsilon)$.

The boundary conditions used to solve Eqs. (1)-(4) dictate no penetration and no slip at the solid wall as well as the balance of shear and normal stresses at the interface. The dimensionless no slip condition at $y=0$ is

$$
u=v=w=0 .
$$

The tangential and normal stress conditions at $y$ $=H(x, z, t)$, are given by

$$
\begin{aligned}
& u_{y}=\sigma_{x}, \quad w_{y}=\sigma_{z}, \\
& p=0+O\left(\epsilon^{2}\right),
\end{aligned}
$$

where the effects of interfacial curvature are neglected since they only enter to $O\left(\epsilon^{2}\right)$ with the scalings chosen. The kinematic condition at the interface described by $v_{s}=d H / d t$, where $v_{s}$ represents the surface velocity in the vertical direction, can be expressed in terms of the fluid flux as: 


$$
H_{t}+\left(H u_{\text {avg }}\right)_{x}+\left(H w_{a v g}\right)_{z}=0
$$

where $u_{a v g}$ and $w_{a v g}$ represent the streamwise and transverse height averaged velocities. Similarly, mass conservation of the insoluble surfactant is expressed by

$$
\Gamma_{t}+\left(\Gamma u_{s}\right)_{x}+\left(\Gamma w_{s}\right)_{z}=\frac{1}{P e_{s}}\left[\Gamma_{x x}+\Gamma_{z z}\right],
$$

where $\Gamma^{*}$ has been scaled by $\Gamma_{m}^{*}$ and $u_{s}$ and $w_{s}$ represent the axial and transverse velocities at $y=H(x, z, t)$. In Eq. (9) the modified surface Peclet number defined by $P e_{s}$ $\equiv\left(U^{*} L_{0}^{*}\right) / \mathscr{D}_{s}^{*}=\left(\Pi_{0}^{*} H_{0}^{*}\right) / \mu^{*} \mathscr{D}_{s}^{*}$, where $\mathscr{D}_{s}^{*}$ is the diffusion coefficient of the surfactant along the interface, represents the ratio of surfactant transport by Marangoni convection to that by surface diffusion. In typical applications, the mass transport by Marangoni convection far exceeds that by surface diffusion. For typical experimental values of $\Pi_{0}^{*}$ $=40 \mathrm{dyn} / \mathrm{cm}, \quad H_{0}^{*}=10^{-3} \mathrm{~cm}, \quad \mu=1 \mathrm{cp}$ and $\mathscr{D}_{s}^{*} \simeq 10^{-5}$ $\mathrm{cm}^{2} / \mathrm{s}, P e_{s} \simeq 10^{5}$. In what follows, we therefore concentrate on flow induced strictly by the balance of viscous and Marangoni terms and treat all other mechanisms as subdominant.

The dimensionless axial and transverse velocity fields are obtained by integrating Eqs. (2) and (4) subject to the boundary conditions in Eqs. (5)-(7):

$$
u(x, y, z)=\sigma_{x} y, \quad w(x, y, z)=\sigma_{z} y .
$$

Substitution of Eq. (10) into Eqs. (8) and (9) in the limit of infinite surface Peclet number yields the two important evolution equations for $H(x, z, t)$ and $\Gamma(x, z, t)$, namely,

$$
\begin{aligned}
& H_{t}+\frac{1}{2}\left(H^{2} \sigma_{\Gamma} \Gamma_{x}\right)_{x}+\frac{1}{2}\left(H^{2} \sigma_{\Gamma} \Gamma_{z}\right)_{z}=0, \\
& \Gamma_{t}+\left(\Gamma H \sigma_{\Gamma} \Gamma_{x}\right)_{x}+\left(\Gamma H \sigma_{\Gamma} \Gamma_{z}\right)_{z}=0 .
\end{aligned}
$$

A constitutive equation of state relating $\sigma$ to $\Gamma$ is required to close this pair of equations. The simplest such relation, valid in the dilute concentration limit, is given by

$$
\sigma(\Gamma)=1-\Gamma .
$$

This linear equation of state approximates the expanded surfactant monolayer as an ideal gas. ${ }^{13}$ When extending these calculations to higher surfactant concentrations, a nonlinear equation of state is required. ${ }^{15}$

\section{B. Self-similar solutions}

Equations (11) and (12) describe the Marangoni driven spreading of an insoluble surfactant monolayer along the surface of a thin liquid film. Substitution of Eq. (13) into Eqs. (11) and (12) yields the evolution equations governing the base state variables, $H_{0}(x, t)$ and $\Gamma_{0}(x, t)$, for onedimensional rectilinear spreading

$$
\begin{aligned}
& H_{0 t}-\frac{1}{2}\left(H_{0}^{2} \Gamma_{0 x}\right)_{x}=0, \\
& \Gamma_{0 t}-\left(\Gamma_{0} H_{0} \Gamma_{0 x}\right)_{x}=0 .
\end{aligned}
$$

In seeking similarity solutions which require global mass conservation (so called similarity solutions of the first kind), we determine the solutions $H_{0}$ and $\Gamma_{0}$ subject to the constraint

$$
M(t)=\int_{0}^{R(t)} \Gamma_{0} d x=M_{0} t^{\gamma},
$$

where $R(t)$ represents the extent of surfactant contamination in time $t$. The choice $\gamma=0$ corresponds to a finite amount of deposited surfactant while $\gamma>0$ corresponds to a reservoir which supplies surfactant to the spreading film at a rate of $t^{\gamma}$. Scale transformations which allow solutions of self-similar form are

$$
\xi=\frac{x}{R(t)}, \quad \Gamma_{0}(x, t)=\frac{g(\xi)}{f(t)}, \quad H_{0}(x, t)=h(\xi) .
$$

Self-similarity requires that $t^{\gamma}=R f^{-1}$, which reduces Eq. (16) to

$$
M_{0}=\int_{0}^{1} g(\xi) d \xi .
$$

These variable transformations convert Eqs. (14) and (15) to

$$
\begin{aligned}
& \lambda_{1} \xi h_{\xi}+\frac{1}{2}\left(h^{2} g_{\xi}\right)_{\xi}=0, \\
& \lambda_{1} \xi g_{\xi}+\lambda_{2} g+\left(g h g_{\xi}\right)_{\xi}=0,
\end{aligned}
$$

where all the explicit time dependence is clustered in the parameters $\lambda_{1}=f R \dot{R}$ and $\lambda_{2}=\dot{f} R^{2}$. Eliminating all the explicit time dependence produces the scaling functions

$$
\begin{aligned}
& R(t)=\left[\lambda_{1} \frac{3}{(1+\gamma)}\right]^{1 / 3} t^{(1+\gamma) / 3}, \\
& f(t)=\left[\lambda_{1} \frac{3}{(1+\gamma)}\right]^{1 / 3} t^{(1-2 \gamma) / 3}, \\
& \lambda_{2}=\lambda_{1}\left[\frac{1-2 \gamma}{1+\gamma}\right] .
\end{aligned}
$$

The extent of spreading for a finite amount of surfactant $(\gamma$ $=0)$ reduces to the solution $R(t)=\left(3 \lambda_{1} t\right)^{1 / 3}$ for rectilinear geometry as previously shown. ${ }^{10,11,16,17}$ The case $\gamma=1 / 2$ yields the spreading behavior $R(t) \sim t^{1 / 2}$, whose base flow solutions ${ }^{18}$ and stability characteristics ${ }^{9}$ have frequently been discussed in the literature.

Equations (19) and (20), which describe the film thickness and surface concentration profiles, cannot be integrated exactly for arbitrary values of $\lambda_{1}$ and $\lambda_{2}$. Simple analytical solutions exist for the choice $\gamma=0$ which determines that $\lambda_{2}=\lambda_{1}$. In this work we focus strictly on the spreading behavior for the case $\gamma=0$. This choice reduces Eq. (20) to the form

$$
\left(\lambda_{1} \xi g+\xi g h g_{\xi}\right)_{\xi}=0 .
$$

The solutions to Eqs. (19) and (24) for the film thickness and concentration profiles require one boundary condition for $h(\xi)$ and two boundary conditions for $g(\xi)$. The two conditions for $g(\xi)$ are derived from consideration of the spreading behavior near $\xi=1$. For quiescent conditions to be recovered far downstream, the surfactant concentration must vanish ahead of $\xi=1$ such that $g(\xi \geqslant 1)=0$. Integration of Eq. (24) across the boundary $\xi=1$ yields the jump condition at the advancing front which determines the second boundary condition on $g$, namely, 


$$
g_{\xi}\left(\xi=1^{-}\right)=-\frac{\lambda_{1}}{h\left(\xi=1^{-}\right)} .
$$

This relation represents the magnitude of the surfactant concentration gradient which develops at the front of a film that has spread to a distance $R(t)=\left(3 \lambda_{1} t\right)^{1 / 3}$. The concentration gradient at this leading edge requires knowledge of the local film thickness and the mass of surfactant available for spreading. Full integration of Eq. (24) yields $h g_{\xi}=-\lambda_{1} \xi$ which, when substituted into Eq. (19), gives

$$
\begin{aligned}
& h(\xi)=A \xi, \\
& g(\xi)=-\frac{\lambda_{1}}{A} \xi+B,
\end{aligned}
$$

where $A=h\left(\xi=1^{-}\right)$and $B=\lambda_{1} / h\left(\xi=1^{-}\right)$. The parameter $\lambda_{1}$ is determined by substituting Eq. (27) into Eq. (18) to give

$$
\lambda_{1}=2 h\left(\xi=1^{-}\right) M_{0} .
$$

What remains to be calculated is the film thickness at the leading edge, $h\left(\xi=1^{-}\right)$, which is evaluated by requiring that the total spreading fluid volume remain constant. Marangoni stresses shear the liquid film and create a linear film profile according to Eq. (26), which when integrated must yield the initial fluid volume $V_{0}=1$. The film thickness is found to achieve a maximum value of $h\left(\xi=1^{-}\right)=2$ which is twice the film height of the initial quiescent film.

The self-similar base flow solutions for the spreading of a finite amount of surfactant are therefore

$$
h(\xi)=2 \xi \quad \text { and } \quad g(\xi)=\left(\lambda_{1} / 2\right)(1-\xi) .
$$

With $\lambda_{1}=1 / 3$, Eq. (29) reduces to the similarity solutions first derived by Jensen and Grotberg. ${ }^{10}$ The dimensionless surface velocity is proportional to $-h g_{\xi}=\lambda_{1} \xi$, which describes the simple shear flow of a Marangoni driven film in the lubrication approximation. It can be shown that the surface velocity in axisymmetric flow is smaller by a factor of $\pi$ than in rectilinear flow due to the fact that the same amount of surfactant must disperse over an ever increasing area producing overall smaller gradients in concentration. We examine the stability of the spreading solutions in rectilinear geometry since it affords the largest driving force for spreading and is therefore expected to be most vulnerable to perturbations.

\section{LINEAR STABILITY ANALYSIS}

\section{A. Perturbation equations}

We have constructed simple analytic forms for the film thickness and concentration profiles in the limit that the spreading process has occurred for a sufficiently long time such that all memory of initial conditions is lost. We now examine the linear stability of these self-similar profiles. Introduction of a two-dimensional perturbation into Eqs. (11) and (12) yields

$$
\begin{aligned}
& \widetilde{h}_{t}=\frac{1}{2}\left(H_{0}^{2} \widetilde{\Gamma}_{x}+2 H_{0} \Gamma_{0 x} \widetilde{h}\right)_{x}+\frac{1}{2} H_{0}^{2} \widetilde{\Gamma}_{z z}, \\
& \widetilde{\Gamma}_{t}=\left(\Gamma_{0} \Gamma_{0 x} \widetilde{h}+H_{0} \Gamma_{0 x} \widetilde{\Gamma}+\Gamma_{0} H_{0} \widetilde{\Gamma}_{x}\right)_{x}+\Gamma_{0} H_{0} \widetilde{\Gamma}_{z z},
\end{aligned}
$$

where the quantities decorated with "tilde" represent perturbations from the base state. Since none of the coefficients in Eqs. (30) and (31) depend explicitly on the transverse coordinate $z$, the perturbation is separable in this coordinate and can be Fourier decomposed into the form

$$
(\widetilde{h}, \widetilde{\Gamma})(x, z, t)=(\widetilde{H}, \widetilde{G})(x, t) e^{i q z},
$$

where $q$ represents the disturbance wavenumber. Substitution of Eq. (32) into Eqs. (30) and (31) yields two coupled partial differential equations that govern the evolution of an applied disturbance

$$
\begin{aligned}
& \widetilde{H}_{t}=\frac{1}{2}\left(H_{0}^{2} \widetilde{G}_{x}+2 H_{0} \Gamma_{0 x} \widetilde{H}\right)_{x}-\frac{q^{2}}{2} H_{0}^{2} \widetilde{G}, \\
& \widetilde{G}_{t}=\left(\Gamma_{0} \Gamma_{0 x} \widetilde{H}+H_{0} \Gamma_{0 x} \widetilde{G}+\Gamma_{0} H_{0} \widetilde{G}_{x}\right)_{x}-q^{2} \Gamma_{0} H_{0} \widetilde{G} .
\end{aligned}
$$

We seek once again solutions of self similar form. Since the applied perturbations will not necessarily evolve on the same time scale as the base state, we describe the disturbance functions by the following transformations:

$$
\widetilde{H}(x, t)=\Psi(\xi, t), \quad \widetilde{G}(x, t)=\frac{\Phi(\xi, t)}{R(t)},
$$

where the scaling of $\Phi(\xi, t)$ by $R(t)$ enforces self-similarity. Other general scaling forms for $\widetilde{G}(x, t)$ will not yield a solvable set of equations without some additional external closure relation. Equations (33) and (34) reduce to the form

$$
\begin{aligned}
\Psi_{t}= & \frac{\dot{R}}{R} \xi \Psi_{\xi}+\frac{1}{2 R^{3}}\left[\left(h^{2} \Phi_{\xi}+2 h g_{\xi} \Psi\right)_{\xi}-\left(q^{2} R^{2}\right) h^{2} \Phi\right], \\
\Phi_{t}= & \frac{\dot{R}}{R}(\xi \Phi)_{\xi}+\frac{1}{R^{3}}\left[\left(g g_{\xi} \Psi+h g_{\xi} \Phi+h g \Phi_{\xi}\right)_{\xi}\right. \\
& \left.-\left(q^{2} R^{2}\right) h g \Phi\right],
\end{aligned}
$$

where $R=t^{1 / 3}$. Without loss of generality, the coefficient of $R$ in Eq. (21) has been set to unity by the choice $\lambda_{1}=1 / 3$. Other choices of $\lambda_{1}$ can simply be absorbed into the rescalings in Eq. (41) to yield the same final result.

\section{B. Quasi-steady-state approximation}

The quasi-steady state approximation (QSSA) assumes that the rate of change of disturbances far exceeds the rate of change of the base state. Inspection of the self-similar base state reveals that the rate of change of $h(\xi)$ and $g(\xi)$ decreases as $t^{-4 / 3}$ in rectilinear geometry. We can therefore assume there exists some time, $t_{q s}$, sufficiently far from $t$ $=0$, beyond which the base state profiles can be regarded as stationary in the variable $\xi$. Within this approximation, the temporal evolution which appears in the coefficients of Eqs. (36) and (37) through the variable $R(t)$ is effectively frozen at $R\left(t_{q s}\right)$. Since the base state then only depends on $\xi$, the coefficients of the two coupled equations are no longer timedependent and the solutions to $\Psi$ and $\Phi$ are separable in time. As a result, $\Psi$ and $\Phi$ assume the form

$$
(\Psi, \Phi)(\xi, t)=e^{\widetilde{\sigma}\left(q, t_{q s}\right) t}(\psi, \phi)\left(\xi, t_{q s}\right),
$$


where $\widetilde{\sigma}$ is the quasi-static growth constant that depends parametrically on $q$ and $t_{q s}$. Substitution of this expanded form into Eqs. (36) and (37) gives

$$
\begin{aligned}
\widetilde{\sigma} \psi= & \frac{\dot{R}\left(t_{q s}\right)}{R\left(t_{q s}\right)} \xi \psi_{\xi}+\frac{1}{2 R\left(t_{q s}\right)^{3}} \\
& \times\left[\left(h^{2} \phi_{\xi}+2 h g_{\xi} \psi\right)_{\xi}-\left(q^{2} R^{2}\right) h^{2} \phi\right]
\end{aligned}
$$

and

$$
\begin{aligned}
\widetilde{\sigma} \phi= & \frac{\dot{R}\left(t_{q s}\right)}{R\left(t_{q s}\right)}(\xi \phi)_{\xi}+\frac{1}{R\left(t_{q s}\right)^{3}} \\
& \times\left[\left(g g_{\xi} \psi+h g_{\xi} \phi+h g \phi_{\xi}\right)_{\xi}-\left(q^{2} R^{2}\right) h g \phi\right] .
\end{aligned}
$$

We replace the base flow profiles, $h$ and $g$, by the expressions derived in Eq. (29) and effect the following three variable changes

$$
\widetilde{\sigma} R^{3}\left(t_{q s}\right) \rightarrow \sigma, \quad q^{2} R^{2}\left(t_{q s}\right) \rightarrow K^{2}, \quad \frac{\psi}{3} \rightarrow \psi .
$$

With these substitutions, Eqs. (39) and (40) reduce to

$$
\begin{aligned}
& \sigma \psi=\left(2 \xi^{2} \phi_{\xi}\right)_{\xi}-\psi-2 K^{2} \xi^{2} \phi, \\
& \sigma \phi=\left(\xi(1-\xi) \phi_{\xi}-\frac{1}{4}(1-\xi) \psi\right)_{\xi}-K^{2} \xi(1-\xi) \phi .
\end{aligned}
$$

Since Eq. (42) determines an equation for $\psi(\xi)$, it can be substituted into Eq. (43) to yield a single third order equation for $\phi(\xi)$, namely,

$$
\begin{aligned}
& \frac{1}{2} \xi^{2}(1-\xi) \phi_{\xi \xi \xi}+\xi\left(2-\frac{5}{2} \xi-(\sigma+1)(1-\xi)\right) \phi_{\xi \xi} \\
& \quad+\left(1-2 \xi-\frac{1}{2} K^{2} \xi^{2}(1-\xi)-(\sigma+1)(1-2 \xi)\right) \phi_{\xi} \\
& \quad+\left(\sigma(\sigma+1)-K^{2} \xi\left(1-\frac{3}{2} \xi-(\sigma+1)(1-\xi)\right)\right) \phi=0 .
\end{aligned}
$$

The quasi-static growth constant $\sigma$ represents the eigenvalue of this linear ordinary differential equation. The real part of the eigenvalue, $\sigma_{r}$, determines the stability of the system. We seek the eigenmode corresponding to the root with the largest real part which signals the fastest growing unstable mode.

\section{Solution procedure}

Equation (44) requires a numerical solution but the procedure is complicated by the existence of two regular singular points at the end points of the domain of integration, namely, $\xi=0$ and $\xi=1$. To investigate the behavior of Eq. (44) in the vicinity of these points, we employ an expansion suggested by the method of Frobenius

$$
\phi(\xi)=\left(\xi-\xi_{j}\right)^{\alpha_{j}} \sum_{i=0}^{\infty} a_{i}\left(\xi-\xi_{j}\right)^{i}, \quad j=1,2,
$$

where $\xi_{1}=0$ and $\xi_{2}=1$. Solution of the indicial equation corresponding to the term $i=0$ yields the solutions to the prefactor exponent $\alpha_{1}=0,0,2 \sigma+1$ and $\alpha_{2}=0,1,3$. We focus on the value $\sigma_{1}=2 \sigma+1<0$ which produces an unbounded solution $\phi(\xi)$ as $\xi \rightarrow 0$. This singular behavior near the origin must be factored out from Eq. (44) before seeking numerical solutions. The eigenfunction $\phi(\xi)$ is therefore redefined to be

$$
\phi(\xi)=\xi^{2 \sigma+1} Y(\xi)
$$

to eliminate any numerical instabilities. Re-expressing Eq. (44) in terms of the well behaved function $Y(\xi)$ gives

$$
Y_{\xi \xi \xi}=-\frac{b(\xi) Y_{\xi \xi}+c(\xi) Y_{\xi}+d(\xi) Y}{a(\xi)},
$$

where

$$
\begin{aligned}
& a(\xi)=\xi^{2}(1-\xi), \\
& b(\xi)=\xi[5-6 \xi+4 \sigma(1-\xi)], \\
& c(\xi)=4(\sigma+1)^{2}-2 \xi(\sigma+1)(2 \sigma+3)-K^{2} \xi^{2}(1-\xi),
\end{aligned}
$$

and

$$
d(\xi)=2 \sigma(\sigma+1)-K^{2} \xi(1-2 \xi) .
$$

Equation (47) is solved subject to the condition that $Y(\xi)$ be regular at the boundaries. This condition allows a Taylor series expansion of the form

$$
Y(\xi)=\sum_{i=0}^{\infty} b_{i}\left(\xi-\xi_{j}\right)^{i}, \quad j=1,2,
$$

which when substituted into Eq. (47) and evaluated at the boundary points $\xi=0,1$ yields the boundary conditions

$$
\begin{aligned}
& Y_{\xi}(0)=-\frac{\sigma}{2(\sigma+1)} Y(0), \\
& Y_{\xi \xi}(0)=\frac{K^{2}-\sigma(\sigma+3)}{(2 \sigma+3)^{2}} Y(0), \\
& Y_{\xi \xi}(1)=-2(\sigma+1) Y_{\xi}(1)+\left[2 \sigma(\sigma+1)+K^{2}\right] Y(1) .
\end{aligned}
$$

Since Eq. (47) is linear in $Y(\xi)$, the solutions are only known to within a multiplicative constant. For convenience, the solutions are normalized by the choice $Y(0)=1$.

\section{Analytical solution for $K=0$ and $\sigma=0$}

Equation (47) admits an analytical solution for the case $K \rightarrow 0$ and $\sigma=0$. In this infinite wavelength limit, the third order differential equation reduces to the simplified form

$$
\left[\xi(1-\xi) \phi_{\xi}^{(0)}-\frac{(1-\xi)}{2}\left(\xi^{2} \phi_{\epsilon}^{(0)}\right)_{\xi}\right]_{\xi}=0
$$

where the superscript (0) denotes the solution for the neutrally stable state. A single integration yields the equation $\xi^{2}(1-\xi) \phi_{\xi \xi}^{(0)}=c_{1}$ where $c_{1}$ is a constant. Two further integrations yield a solution of the form

$$
\phi^{(0)}=c_{3}+c_{2} \xi+c_{1}(1-\xi) \ln \left(\frac{1-\xi}{\xi}\right),
$$


where $c_{2}$ and $c_{3}$ are two more constants of integration to be determined from boundary conditions. In this same limit, Eq. (42) can be used to derive the corresponding solution for the perturbed film thickness, $\psi^{(0)}$ :

$$
\psi^{(0)}=\left(2 \xi^{2} \phi_{\xi}^{(0)}\right)_{\xi}=4 c_{2} \xi+2 c_{1}\left(2 \xi \ln \frac{\xi}{1-\xi}-\frac{\xi}{1-\xi}\right) .
$$

Applying the constraint that both functions $\psi^{(0)}$ and $\phi^{(0)}$ be regular at the endpoints $\xi=0$ and $\xi=1$ necessitates the choice $c_{1}=0$. Furthermore, since all singular behavior has been extracted from $\phi(\xi)$ through Eq. (46) such that $\phi^{(0)}(\xi)=\xi Y(\xi)$, it follows that $\phi^{(0)}(0)=0$ since $Y(\xi)$ is a regular and well behaved function in $\xi$. According to Eq. (53) then, $c_{3}=0$ and the analytical solutions are simply of the form $\phi^{(0)}(\xi)=c_{2} \xi$ and $\psi^{(0)}(\xi)=4 c_{2} \xi$. Because the governing equations are linear, the solutions are only known to within an overall constant which for convenience is chosen to be $c_{2}=1$ such that

$$
\begin{aligned}
& \phi^{(0)}=\xi, \\
& \psi^{(0)}=4 \xi .
\end{aligned}
$$

These analytical solutions provide a numerical check on the shooting technique used to solve $Y(\xi)$.

\section{RESULTS AND DISCUSSION}

\section{A. Numerical solutions}

The third order differential equation for $Y(\xi)$ shown in Eq. (47) has been converted into an eigenvalue problem which was solved with a standard shooting technique. The numerical solutions were constructed by shooting away from $\xi=0$ and $\xi=1$ and applying the three boundary conditions some small distance $\Delta \xi$ away from each endpoint. One must shoot away from these two endpoints since the denominator in Eq. (47) vanishes at $\xi=0$ and 1 . The solutions for $Y(\xi)$ are insensitive to the choice of $\Delta \xi$ for $\Delta \xi \sim O\left(10^{-4}\right)$ or smaller. Simultaneously shooting away from both endpoints with the requirement that $Y, Y_{\xi}$ and $Y_{\xi \xi}$ be continuous at the midpoint $\xi=0.5$ yields the condition about which the values of $\sigma$ are iterated to find the appropriate eigenvalue. Different matching points in the interval yielded the same solutions. A fourth order Runge-Kutta algorithm was used as the initial value solver and the step size was adjusted to ensure the continuity requirements at $\xi=0.5$. Solutions to $Y(\xi)$ and $\sigma$ were substituted into Eq. (46) and Eq. (42) to reconstruct the full eigenfunctions $\phi(\xi)$ and $\psi(\xi)$.

In Fig. 3(a) is shown the dispersion curve, $\sigma\left(K^{2}\right)$, generated from the shooting method. This curve was constructed by solving Eq. (47) for different values of wavenumber $K^{2}$ varied in small increments of the order of $\Delta K^{2}=0.002$. The eigenfunction solutions varied smoothly as the wavenumber was increased indicating no sudden crossings from one branch of $\sigma$ to another. In an effort to uncover any positive roots or any negative roots lying above the solution shown, the initial guesses for $\sigma$ were systematically varied in magnitude. For instance, in searching for the roots lying close to the point $K=0$, we tried initial guesses for $\sigma$ ranging from 0 to 2 in increments of $10^{-4}$. In all cases, the solution always
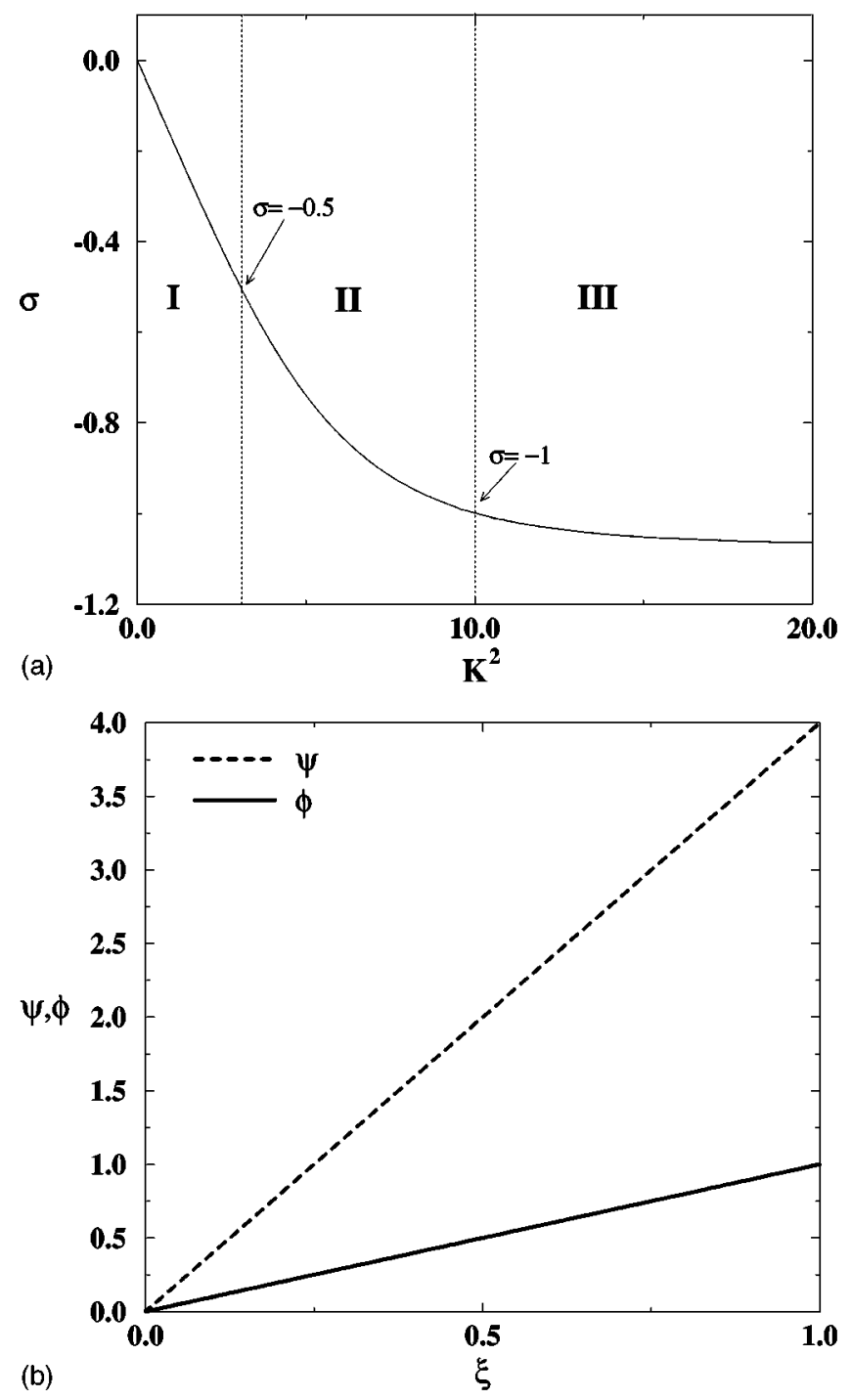

FIG. 3. (a) Dispersion relation $\sigma\left(K^{2}\right)$. (b) Neutrally stable eigenfunctions $(\sigma=0) \psi$ and $\phi$ for $K^{2}=0$.

(i) converged to the root shown in Fig. 3(a), (ii) converged to an additional branch that exhibits a maximum value of $\sigma=$ -1.327 at $K=0$ and continues decreasing, or (iii) never converged. This second branch is of little interest from a stability standpoint and was not studied further. We were unable to find convergent solutions for initial guesses $\sigma>2$ irregardless of the wavenumber. The solution plotted in Fig. 3(a) therefore appears to belong to a single continuous branch.

\section{B. Characteristics of the dispersion curve and associated eigenfunctions}

The absence of any positive roots to the coupled linear system of equations indicates that within the quasi-steady state approximation, the self-similar base flow profiles, $h(\xi)=2 \xi$ and $g(\xi)=\lambda_{1}(1-\xi) / 2$, are linearly stable to infinitesimal perturbations. It is difficult to understand this result on purely physical grounds especially since the modelling includes no explicit stabilizing mechanisms like capillarity or surface diffusion. The source of the stabilizing mechanism can only be traced to the transverse Marangoni 

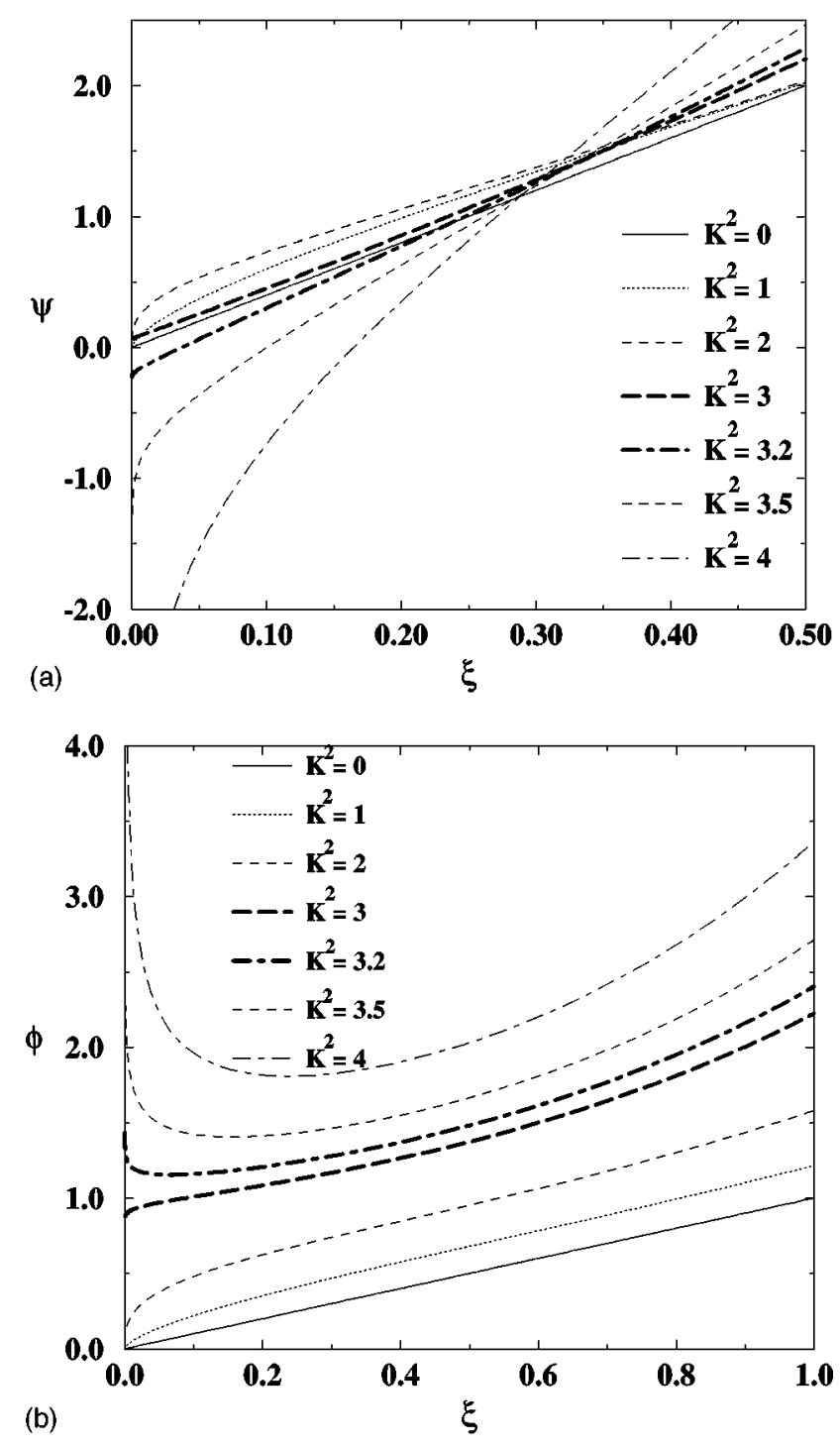

FIG. 4. Eigenfunctions $\psi$ and $\phi$ near $K^{2}=3$.

convection established by local disturbances. To understand more fully exactly how the transverse Marangoni contributions can counteract disturbances of any wavelength, we investigate the shape of the eigenfunctions $\psi$ and $\phi$ for different regimes in $K$ space. The dispersion curve shown in Fig. 3(a) divides itself naturally into three regions labeled I, II, and III, each reflecting a somewhat different character in $\psi$ and $\phi$. Region I spans the range $0 \leqslant K^{2} \leqslant 3.2$, region II the range $3.2 \lesssim K^{2} \lesssim 10$, and region III the range $K^{2} \gtrsim 10$.

We focus first on the eigenfunction solutions for infinitely long wavelength disturbances, $K=0$, plotted in Fig. 3(b). The shooting technique correctly reproduces the analytical solutions derived in Eqs. (55) and (56) for $\sigma=0$. These neutrally stable solutions increase linearly throughout the domain of integration $0 \leqslant \xi \leqslant 1$, with perturbations in the dimensionless film thickness achieving a four fold increase over perturbations in the dimensionless surfactant concentration. This result sheds light on the long wavelength approximation used in a previous stability calculation ${ }^{9}$ in which the amplitude of the disturbance film thickness was assumed to

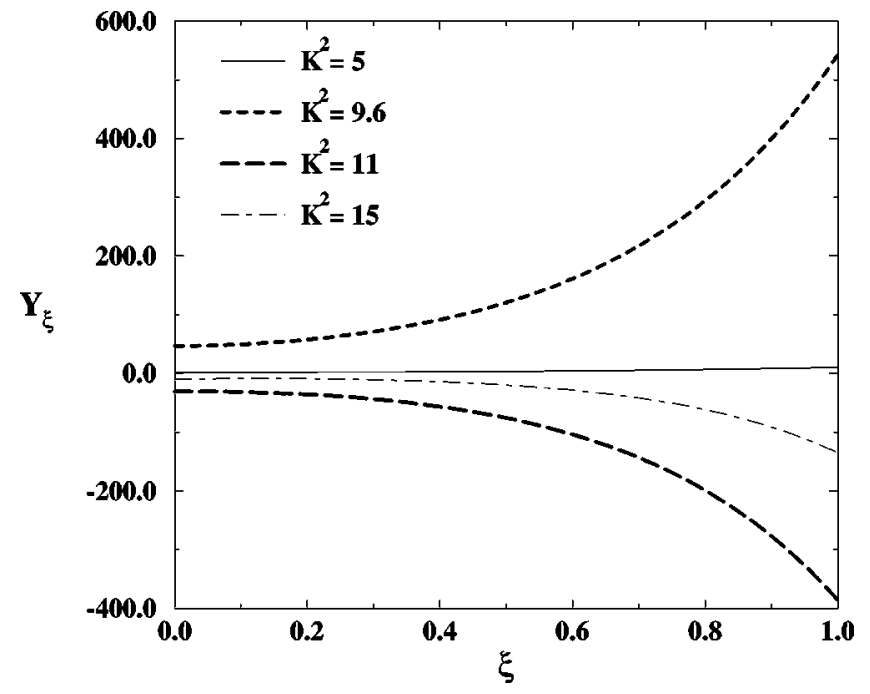

FIG. 5. Behavior of $Y_{\xi}(\xi)$ near $K^{2}=10$.

be much smaller than the amplitude of the disturbance concentration. These earlier studies predicted linearly unstable flow. Although the base flow profiles being studied are different [i.e., $R(t) \sim t^{1 / 3}$ versus $R(t) \sim t^{1 / 2}$ ], it now appears that allowing both the surfactant concentration and the film thickness to undergo disturbances self-consistently produces overall stable flow. Spreading behavior governed by $R(t) \sim t^{1 / 2}$ cannot be treated analytically as simply as the $R(t) \sim t^{1 / 3}$ case. At present there is no direct comparison one can make between these two stability calculations.

It is interesting to note that in other thin film spreading problems, like the coating of a dry substrate by a liquid film subject to gravitational forces, ${ }^{9}$ centrifugal forces ${ }^{19}$ or surface shear stresses,$^{20}$ the linearized form of the equation of motion for the film thickness is translationally invariant in the streamwise direction. This symmetry dictates that the eigenfunction solution for $\sigma=0$ be directly proportional to the first derivative of the base flow profile. In contrast, the linearized equations of motion for Marangoni driven flow contain explicit dependence on the streamwise coordinate $\xi$, as evident in Eqs. (42) and (43). The eigenfunction solutions are therefore not neatly related to $h_{\xi}$ and $g_{\xi}$. Had this explicit dependence on $\xi$ not been present, the associated eigenfunctions would be flat throughout the interval of integration unlike the actual monotonically increasing ramps plotted in Fig. 3(b).

For finite wavenumbers, the individual shape of the eigenfunctions $\psi$ and $\phi$ and their relation to each other changes significantly in each of the three regions. In Fig. 4 is shown the eigenfunctions for wavenumbers ranging from 0 $\leqslant K^{2} \leqslant 4$.0. In order to magnify certain features near $K=0$, the function $\psi$ is only plotted in the range $0 \leqslant \xi \leqslant 0.5$. The solutions change character upon traversing the value $K^{2}$ $\approx 3.2$. With increasing wavenumber, the functions $\psi$ and $\phi$ increase in absolute magnitude for small $\xi$ and develop strong curvature near the boundary points $\xi=0$ and $\xi=1$, in contrast to the linear profiles for $K^{2}=0$. Near a value of $K^{2} \approx 3.2$ the function $\psi$ changes sign near the origin and becomes increasingly negative while $\phi$ becomes increasingly 

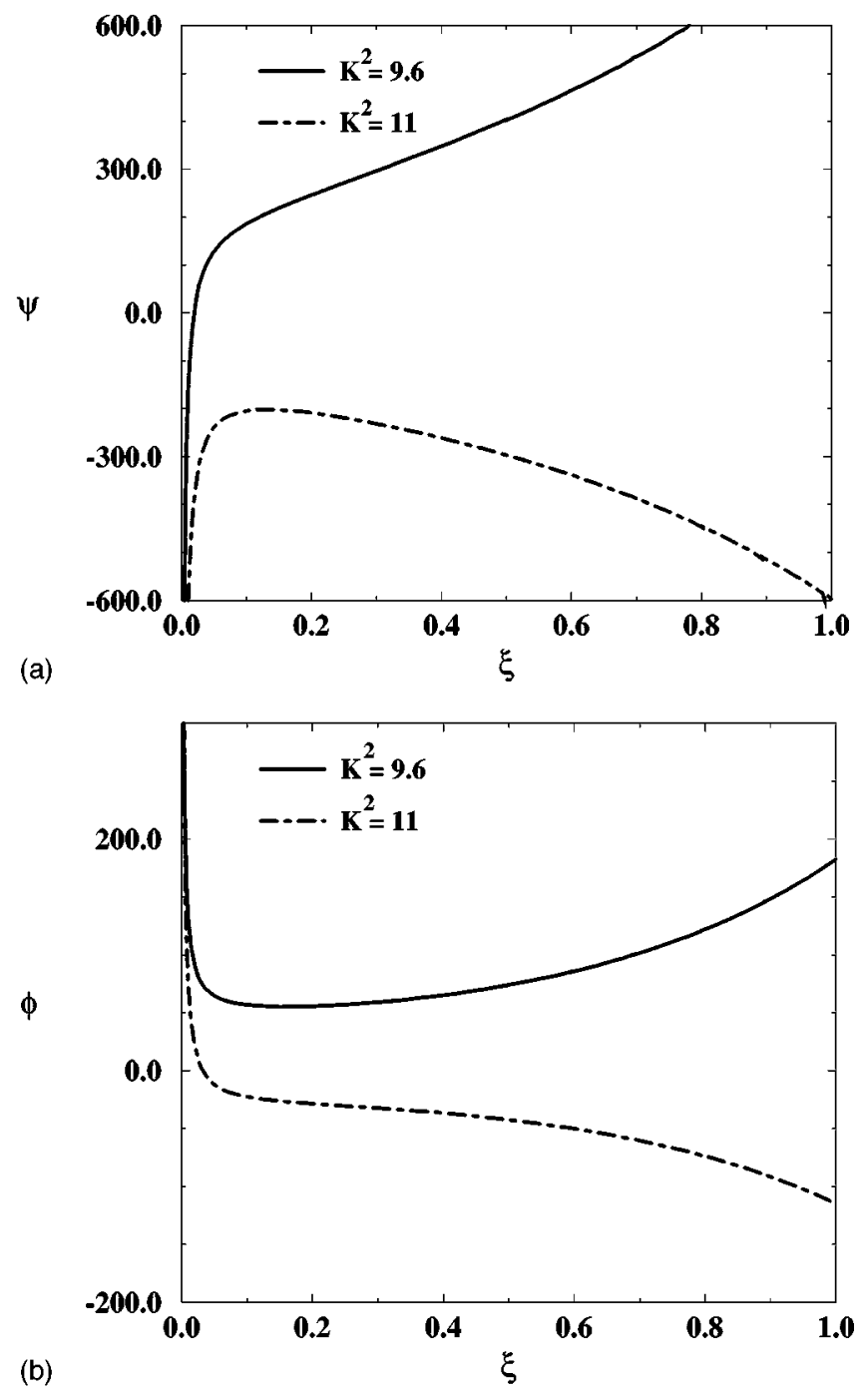

FIG. 6. Eigenfunctions $\psi$ and $\phi$ near $K^{2}=10$.

positive. The value $K^{2} \approx 3.2$ signals a transition in behavior which derives from the Frobenius expansion $\phi$ $\sim \xi^{2 \sigma+1} Y(\xi)$, wherein $\phi$ diverges for values $\sigma<-1 / 2$, or equivalently for wavevectors $K^{2} \geq 3.2$. This singular behavior at the origin creates corresponding singular behavior in $\psi$ as shown in Fig. 4.

Another change in the behavior of the eigenfunctions occurs upon traversing region II into region III as predicted by the boundary condition in Eq. (49) which suffers a pole at $\sigma=-1$. Since the normalization condition was chosen to be $Y(0)=1$, Eq. (49) requires that $Y_{\xi}$ and therefore $\phi(\xi)$ diverge at the origin when $\sigma=-1$, which occurs for $K^{2}$ $\approx 9.8$. In Fig. 5 we show how $Y_{\xi}(\xi)$ changes character for various choices of wavenumber lying on either side of this transition point. As $K^{2}$ increases through this special point, the function $Y_{\xi}(\xi)$ must suddenly change sign from positive to negative values. This change in sign causes a significant change in the behavior of $\psi$ and $\phi$ as demonstrated in Fig. 6. For example, whereas $\psi$ achieves negative values close to the origin for $K^{2}=9.6$ but is positive everywhere else in the domain, the function switches sign and becomes everywhere negative after passing through the transition point.

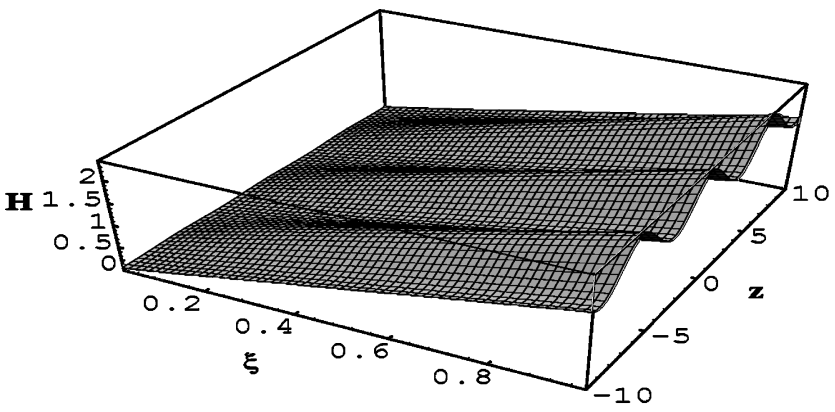

(a)

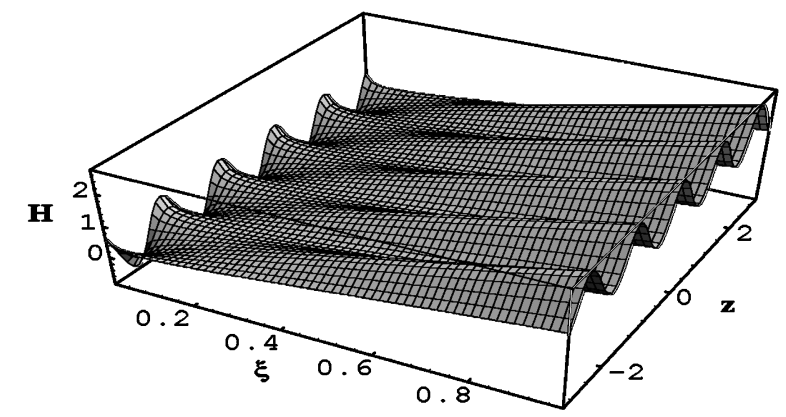

(b)

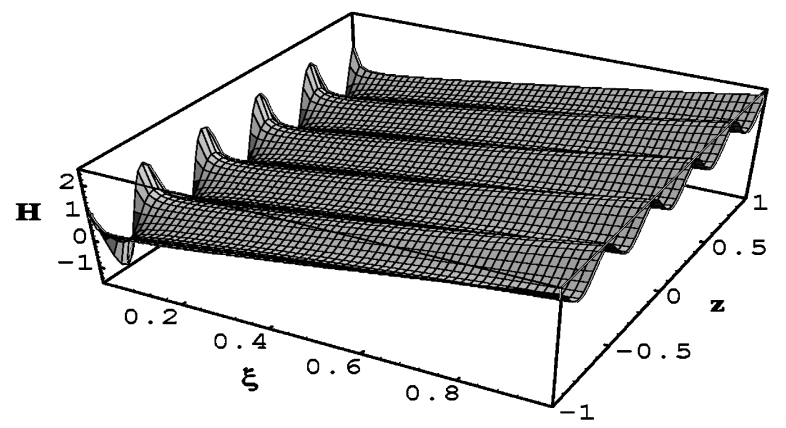

(c)

FIG. 7. Surface plots of the total film thickness: (a) region $\mathrm{I}\left(K^{2}=1\right)$, (b) region II $\left(K^{2}=5\right)$ and (c) region III $\left(K^{2}=15\right)$.

To visualize the overall effect of an infinitesimal disturbance on the flow properties of a film driven by Marangoni forces in each of the regions specified above, we plot in Fig. 7 the complete linearized solution to the film thickness which is a superposition of the base state and the disturbance eigenfunction extended in the $z$ direction. Each figure demonstrates the typical behavior of the film thickness for some choice of wavevector within each of the three regimes delineated in Fig. 3(a). Note that the solutions in Figs. 7(a) and (b) maintain registry in the streamwise direction wherein points of maximum depression at the origin become points of highest elevation at $\xi=1$ and vice versa, whereas the solution in Fig. 7(c) does not. Although the transient disturbances assume the shape of slender fingers or rivulets throughout the domain of spreading, the flows in regions I, II, and III are all linearly stable and decay away exponentially in time. In regions II and III, the magnitude of the perturbations near the origin is significantly larger than the magnitude of the per- 


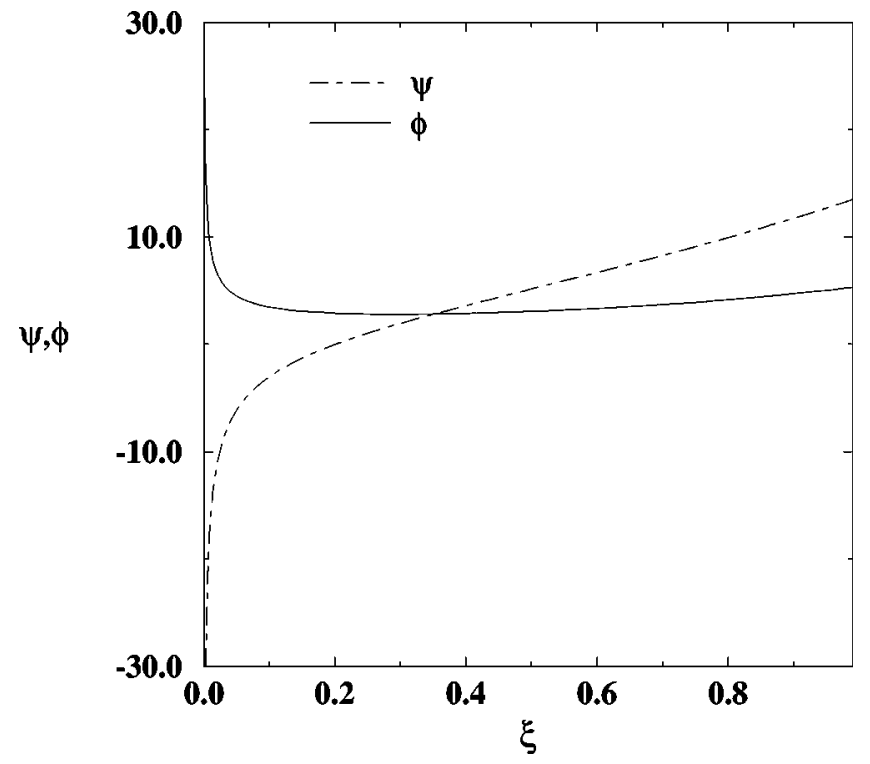

FIG. 8. Illustration of the complementary relation between $\psi$ and $\phi$ for $K^{2}=5$.

turbations near the endpoint $\xi=1$, which is more clearly seen from Figs. 6 and 8.

There exists an interesting complementary relationship between $\psi$ and $\phi$ in regions II and III which could possibly have yielded unstable flow according to the following reasoning. Observe in Fig. 8 that near the origin, where the film thickness suffers appreciable thinning, the liquid mobility will decrease substantially thereby retarding convection of fluid and surfactant in this region. Surfactant accumulates here to create a region of particularly low surface tension as demonstrated by the form of $\phi(\xi)$. Transverse Marangoni stresses will further pull on this film causing it to thin even further. This mechanism should create strong corrugations in film thickness and create "fingered" patterns in the transverse direction. Unfortunately, this complementary behavior between $\psi$ and $\phi$ only exists in a very small region near the origin and is apparently not significant enough to destabilize the flow. In the next section, we present results of an energy analysis to quantify the relative magnitude of stabilizing and destabilizing contributions to the overall flow behavior.

\section{The energy method}

Within our simplified model only Marangoni stresses generated by the presence of surfactant drive the spontaneous spreading process. For the unperturbed 1-D flow, these stresses convect fluid and surfactant downstream rapidly and efficiently. The application of an arbitrary 2-D disturbance creates additional stresses with subsequent transport of fluid and surfactant in the transverse direction. According to the linear stability analysis, this transverse flow successfully dampens disturbances of all wavenumbers. By decomposing the flow into its constituent contributions we can better appreciate the relative scale of streamwise versus transverse mass flux for disturbances of self-similar form.

The mechanical energy generated by an applied perturbation can equivalently be expressed as an inner product of
TABLE I. Physical mechanisms associated with each term in the energy method.

\begin{tabular}{|c|c|c|}
\hline Terms & Expression & Physical mechanism \\
\hline 1 & $\int_{0}^{1}\left[\psi\left(2 \xi^{2} \phi_{\xi}\right)_{\xi}-\psi^{2}\right] d \xi$ & $\begin{array}{l}\text { Marangoni convection of } \\
\text { fluid layer in the } \xi \text { direction. }\end{array}$ \\
\hline 2 & $-2 K^{2} \int_{0}^{1}\left[\xi^{2} \psi \phi\right] d \xi$ & $\begin{array}{l}\text { Marangoni convection of } \\
\text { fluid layer in the } z \text { direction. }\end{array}$ \\
\hline 3 & $\int_{0}^{1}\left[\phi\left(\xi(1-\xi) \phi_{\xi}-\frac{1}{4}(1-\xi) \psi\right)_{\xi}\right] d \xi$ & $\begin{array}{c}\text { Marangoni convection of } \\
\text { surfactant monolayer } \\
\text { in the } \xi \text { direction. }\end{array}$ \\
\hline 4 & $-K^{2} \int_{0}^{1}\left[\xi(1-\xi) \phi^{2}\right] d \xi$ & $\begin{array}{l}\text { Marangoni convection of } \\
\text { surfactant monolayer } \\
\text { in the } z \text { direction. }\end{array}$ \\
\hline
\end{tabular}

the disturbance film thickness or the disturbance concentration according to

$$
\begin{aligned}
& E=\frac{1}{2} \int_{0}^{1} \Psi^{2} d \xi=\frac{1}{2}\langle\Psi, \Psi\rangle, \quad \text { or } \\
& E=\frac{1}{2} \int_{0}^{1} \Phi^{2} d \xi=\frac{1}{2}\langle\Phi, \Phi\rangle .
\end{aligned}
$$

Recasting Eqs. (36) and (37) in compact operator form gives

$$
\Psi_{t}=\tilde{\mathscr{L}}_{1}[\Psi, \Phi]
$$

and

$$
\Phi_{t}=\widetilde{\mathscr{L}}_{2}[\Psi, \Phi],
$$

where the linear operators $\widetilde{\mathscr{L}}_{1}$ and $\widetilde{\mathscr{L}}_{2}$ represent all the terms on the right hand side of Eqs. (36) and (37). The subscript $t$ denotes differentiation with respect to the explicit time dependence. The rate of energy production, $d E / d t=\langle\Psi, \dot{\Psi}\rangle$ $=\langle\Phi, \dot{\Phi}\rangle$, is calculated by taking the inner product of Eq. (59) with $\Psi$ or the inner product of Eq. (60) with $\Phi$ to give

$$
\frac{d E}{d t}=\widetilde{\sigma}\langle\Psi, \Psi\rangle=\left\langle\Psi, \widetilde{\mathscr{B}}_{1}[\Psi, \Phi]\right\rangle,
$$

or

$$
\frac{d E}{d t}=\widetilde{\sigma}\langle\Phi, \Phi\rangle=\left\langle\Phi, \widetilde{\mathscr{L}}_{2}[\Psi, \Phi]\right\rangle .
$$

The normalized dimensionless rate of energy production, $\dot{\mathscr{E}}$ $=(d E / d t) / E$, is therefore calculated to be

$$
\frac{\dot{\mathscr{C}}}{2}=\sigma=\frac{\left\langle\psi, \mathscr{L}_{1}[\psi, \phi]\right\rangle}{\langle\psi, \psi\rangle},
$$

or

$$
\frac{\dot{\mathscr{E}}}{2}=\sigma=\frac{\left\langle\phi, \mathscr{L}_{2}[\psi, \phi]\right\rangle}{\langle\phi, \phi\rangle} .
$$

The terms $\left\langle\psi, \mathscr{L}_{1}[\psi, \phi]\right\rangle$ and $\left\langle\phi, \mathscr{L}_{2}[\psi, \phi]\right\rangle$, where $\mathscr{L}_{1}$ and $\mathscr{L}_{2}$ are the right hand sides of Eqs. (42) and (43), comprise four separate terms shown in Table I. The first two terms represent Marangoni convection of liquid in the streamwise and transverse directions, while the last two terms correspond to Marangoni convection of surfactant in the stream- 


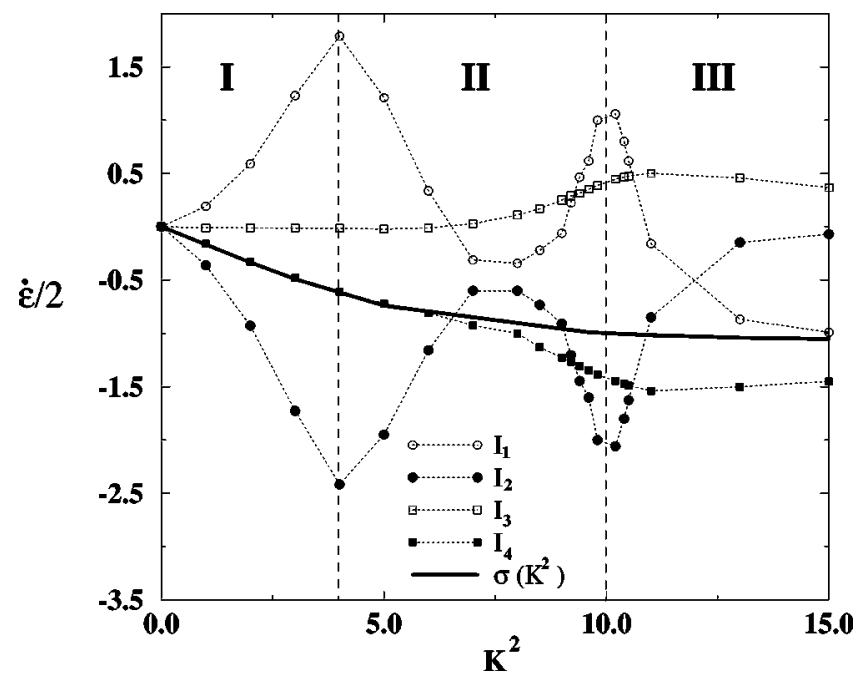

FIG. 9. Variation of $\mathscr{E} / 2$ with $K^{2}$. Vertical dotted lines reflect solutions $\sigma$ $=-0.5$ and $\sigma=-1$.

wise and transverse directions. These terms arise from the coupling of the 1-D spatially inhomogeneous base flows with the applied 2-D disturbance.

Results of energy analysis. The normalized dimensionless rate of energy production for each of the four terms is plotted in Fig. 9, along with their summation, which must exactly equal the value $2 \sigma$ for each wavevector selected. The vertical dotted lines indicate the transition points discussed earlier for which $\sigma=-0.5$ or -1 . Positive integral quantities destabilize the flow while negative integral quantities provide a stabilizing influence. The quantity $I_{i}$ represents the energy integral associated with term $i$ in Table I. Inspection of these different terms automatically reveals which contributions would have to be amplified or minimized significantly to produce positive roots in the dispersion curve $\sigma\left(K^{2}\right)$.

$I_{1}$ exhibits two maxima and $I_{2}$ two minima precisely at the location of the transition points and reflect the change in behavior in $\psi$ and $\phi$ which occurs upon traversal of these points. These changes do not affect the behavior of $I_{3}$ and $I_{4}$ as strongly, although there occurs a slightly larger increase in the amplitude of these terms near the second transition point as compared to the first. This overall behavior is expected since the amplitude of the eigenfunctions corresponding to the film thickness, which affects terms $I_{1}$ and $I_{2}$, is typically much larger than the amplitude of the eigenfunctions associated with the surfactant concentration, which affects terms $I_{3}$ and $I_{4}$. What is clearly noticeable in the figure is that the majority of the energy contributions are weighted toward the negative end of the energy spectrum. Terms $I_{2}$ and $I_{4}$, which reflect Marangoni convection of fluid and surfactant in the transverse direction, are negative for all wavevectors and large enough to offset any destabilizing effects in the streamwise direction.

Further inspection of terms $I_{1}$ and $I_{2}$ associated with the liquid flux reveals that that the eigenfunction pairs for which $\psi \phi<0, \psi \phi_{\xi}>0$ and $\psi \phi_{\xi \xi}>0$ maximize the destabilizing term $I_{1}$ and minimize the stabilizing term $I_{2}$. The eigenfunction solutions for $K^{2} \gtrsim 3.2$ or equivalently for $\sigma<-0.5$ nicely satisfy these inequalities but only in a very limited range about the origin $\xi=0$. Outside this range the inequalities are not satisfied and the contributions to the flow are overall stabilizing. For unstable flow this Marangoni driven system must generate eigenfunction pairs with the complementary nature indicated by the three inequalities in which case it can tilt the energy balance towards the positive values of the energy spectrum. As summarized in the last section, a local decrease in film thickness accompanied by a local increase in surfactant concentration will provide the proper scenario for producing a lateral fingering instability.

What direction might one pursue in order to model a system of equations that can produce and exploit this complementarity between $\psi$ and $\phi$ ? We have shown that the unfavorable results of the stability analysis are directly caused by the linear behavior of the self-similar solutions in Eq. (29) for which an increase in film thickness is accompanied by a decrease in surfactant concentration. All disturbances eventually die away due to the increasing liquid mobility provided by the linearly increasing ramp in film thickness from $0 \leqslant \xi \leqslant 1$. In order to localize disturbances behind the moving front, the film thickness must somewhere suffer a decrease in thickness which will further be aggravated by a consequent increase in surfactant concentration. There is another fluid mechanical problem, namely SaffmanTaylor flow, ${ }^{12}$ for which regions of adverse mobility gradient produce unstable fingering configurations. As an example of this flow, consider the case of a gas penetrating into a viscous liquid sandwiched between two plates of constant separation. The average fluid velocity is $u_{S T}=-\left(b^{2} /\right.$ $12 \mu) d P / d x$, where $b$ is the plate spacing, $\mu$ the liquid viscosity, and $d P / d x$ the local pressure gradient. Since the gaseous phase experiences a decrease in mobility when penetrating into the more viscous medium, it can easily be shown that the front separating the two regions of differing mobility becomes linearly unstable and propagates fingers into the viscous liquid. For the case of a viscous liquid penetrating a gas, the front is stable. We have shown that the average velocity of a thin film sheared by Marangoni stresses is $u_{M}$ $=-(h / 2 \mu) d \Gamma / d x$ wherein the quantity $h / 2 \mu$ can be regarded as the mobility factor. Although in our system the viscosity is constant throughout, a local decrease in the film thickness $h$ can effectively lower the local film mobility. Appealing to this concept of adverse mobility, we describe what other forces can be included in the spreading problem to produce exactly such regions of reduced mobility.

The inclusion of capillarity and surface diffusion into the equations of motion obviates the possibility of finding simple analytic self-similar solutions for the unperturbed flow, a fact which eventually complicates the linear stability analysis. Nonetheless, the method of lines ${ }^{21}$ can be used to solve the equations of motion numerically in the presence of these additional forces, as first discussed by Gaver and Grotberg. ${ }^{22}$ Not only do these forces help smooth numerical instabilities associated with the sharp fronts created by Marangoni stresses alone, but they also change the character of the solutions from simple ramp-like behavior over a finite domain to a more complex, spatially inhomogeneous structure of semi-infinite extent. It may seem that the addition of these 


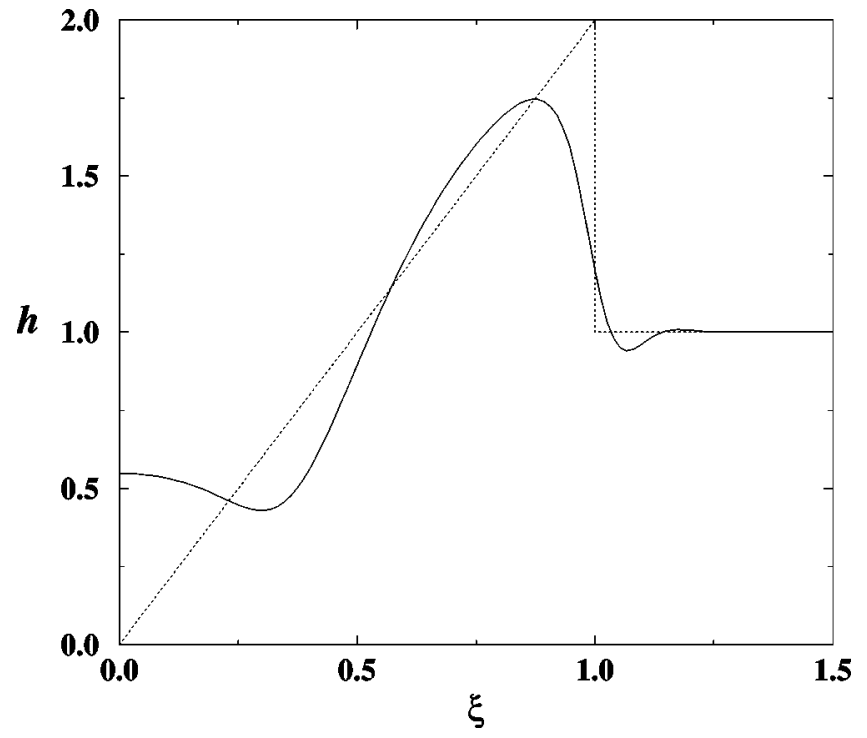

FIG. 10. Film thickness profiles for (i) linear self similar solution governed by Eq. (29) and (ii) numerical solution including capillarity and surface diffusion for the case of $\mathrm{Ca}=10^{-4}, \mathrm{Pe}_{s}=5.10^{3}$ and dimensionless time $t$ $=31$.

two forces will only further stabilize the flow. We propose, however, that the change in character of the form of the solutions produces regions of adverse mobility known to destabilize the flow in the Saffman-Taylor problem. ${ }^{12}$ We have plotted in Fig. 10 the self-similar solution for the dimensionless film thickness from Eq. (29) along with a numerical solution for the film thickness profile with the inclusion of capillarity and surface diffusion. As expected, this numerical profile more closely resembles experimental observations but more importantly, it suffers two regions of adverse mobility gradients, namely the region near the point of surfactant deposition at the left and the region where the sharp advancing front joins the thinner undisturbed clean film at the right. We are presently investigating the linear stability of these numerically generated profiles to uncover if either is vulnerable to finger formation in the transverse direction.

\section{CONCLUSION}

We have investigated within the lubrication approximation the base flow profiles and linear stability for the rectilinear spreading of an insoluble surfactant along a thin liquid film of higher surface tension. In the limit in which the spontaneous spreading is only controlled by Marangoni stresses, the unperturbed profiles for the film thickness and surfactant concentration can be computed analytically. The profiles chosen for study are self-similar solutions of the first kind corresponding to global surfactant mass conservation. In this frame of reference, since the film thickness is a linearly increasing function while the surface concentration is a linearly decreasing one, the velocity field describes a simple shear flow. This flow leads to severe thinning of the fluid layer near the surfactant deposition point and a rapidly advancing rim at the leading front, of thickness twice the undisturbed height. This general shape in film thickness bears close resemblance to experimental observations. ${ }^{2}$
The linear stability of the disturbance equations is calculated within the quasi-steady state approximation (QSSA), which assumes that the rate of change of the base state is much slower than the rate of change of any disturbance. This approximation leads to a coupled eigenvalue problem whose largest real root is identified as the quasi-static growth rate for the most unstable mode. Results obtained from the QSSA analysis determine that the coupled system of equations is linearly stable to 2-D disturbances of all wavenumbers. 3-D visualizations of the complete linearized solution indicate finger like protrusions throughout the spreading domain; however they decay away exponentially in time to restore the film thickness to its original ramp-like profile. The fact that the calculations in rectilinear geometry, which provides the largest impetus for Marangoni driven spreading, yield linearly stable flow strongly suggests that an additional characteristic of the flow must be included in future analyses.

An energy decomposition reveals how Marangoni convection in the transverse direction successfully stabilizes the system against infinitesimal 2-D perturbations. The terms responsible for destabilizing the flow are most dominant in the range $K^{2} \geq 3.2$ but only occur within a narrow range about the origin $\xi=0$. We have identified that eigenfunctions satisfying the inequalities $\psi \phi<0, \psi \phi_{\xi}>0$ and $\psi \phi_{\xi \xi}>0$ over a much larger range in $\xi$ can potentially destabilize the overall flow. We describe a way to enhance the complementary nature of the eigenfunctions reflected in these inequalities by introducing into the equations of motion the additional subdominant forces of capillarity and surface diffusion. The inclusion of these terms changes the shape of the base flow profiles significantly to create two regions where the film thickness thins substantially thereby reducing the flow mobility. In analogy with the Saffman-Taylor problem, ${ }^{12}$ the creation of adverse mobility gradients can possibly destabilize the flow.

There exist other avenues of inquiry regarding the stability of a Marangoni driven spreading film. For example, since the velocity profile for a thin film driven strictly by Marangoni stresses (no capillarity or surface diffusion effective) is an example of a thin film under simple shear, the flow dynamics may turn out to be linearly stable, as shown by Romanov $^{23}$ and others for planar Couette flow. One should then simulate the fully 3-D flows and numerically investigate the flow behavior to finite amplitude disturbances, as presently underway. Also, since the operators $\mathscr{L}_{1}$ and $\mathscr{L}_{2}$ are non self-adjoint, we are simultaneously conducting a transient growth analysis ${ }^{24}$ to determine if certain modes grow sufficiently in the early stages of spreading to activate a large non-linear response. We hope that our present linear stability analysis of the self similar solutions provides a provoking starting point into the stability considerations of Marangoni driven spreading.

\section{ACKNOWLEDGMENTS}

This work was supported by an NSF Research Initiation Award through Grant No. CTS-9409579 and an NSF CAREER award through Grant No. CTS-9624776. The authors would also like to acknowledge interesting discussions with M. Göz, M. Kostin, R. Jackson, S. H. Lam and S. A. Orszag. 
${ }^{1}$ A. Marmur and M. D. Lelah, "The spreading of aqueous surfactant solutions on glass," Chem. Eng. Commun. 13, 133 (1981).

${ }^{2}$ S. M. Troian, X. L. Wu, and S. A. Safran, "Fingering instability in thin wetting films," Phys. Rev. Lett. 62, 1496 (1989).

${ }^{3}$ S. M. Troian, A. Pereira, and O. K. Matar (unpublished).

${ }^{4}$ G. M. Homsy, "Viscous fingering in porous media," Annu. Rev. Fluid Mech. 19, 271 (1987).

${ }^{5}$ S. Zhu, W. G. Miller, L. E. Scriven, and H. T. Davis, "Superspreading of water-silicone surfactant on hydrophobic surfaces," Colloids Surf., A 90, 63 (1994).

${ }^{6} \mathrm{~B}$. Frank and S. Garoff, "Origins of the complex motion of advancing surfactant solutions,' Langmuir 11, 87 (1995).

${ }^{7} \mathrm{~S}$. He and J. Ketterson, "Surfactant driven spreading of a liquid on a vertical surface," Phys. Fluids 7, 2640 (1995).

${ }^{8}$ S. M. Troian, X. L. Wu, and S. A. Safran, in Phase Transitions in Soft Condensed Matter, NATO ASI Series Vol. 211, edited by T. Riste and D. Sherrington (Plenum Press, New York, 1989).

${ }^{9}$ S. M. Troian, E. Herbohlzheimer, and S. A. Safran, "Model for the fingering instability of spreading surfactant drops," Phys. Rev. Lett. 65, 333 (1990).

${ }^{10} \mathrm{O}$. E. Jensen and J. B. Grotberg, "Insoluble surfactant spreading on a thin viscous film: Shock evolution and rupture," J. Fluid Mech. 240, 259 (1992).

${ }^{11} \mathrm{O}$. E. Jensen and J. B. Grotberg, "The spreading of heat or soluble surfactant along a thin liquid film," Phys. Fluids A 5, 58 (1993).

${ }^{12}$ P. G. Saffman and G. I. Taylor, "The penetration of a fluid into a porous medium or Hele-Shaw cell containing a more viscous liquid," Proc. R. Soc. London, Ser. A 245, 312 (1958).
${ }^{13}$ A. W. Adamson, in Physical Chemistry of Surfaces (Wiley, New York, 1990), Chap. 3, pp. 87-88.

${ }^{14}$ Requiring $H^{*} \ll \epsilon L_{0}^{*}$ is more restrictive than necessary. Upon deposition of surfactant, the liquid film is strongly sheared and rapidly thins to satisfy the lubrication approximation. The derivation for the spreading process is therefore valid even for systems that do not strictly obey the inequality.

${ }^{15}$ A. Sheludko, Colloid Chemistry (Elsevier, New York, 1966).

${ }^{16}$ F. F. Espinosa, "Spreading of surfactant in a pulmonary airway," Adv. Bioeng. 20, 571 (1991).

${ }^{17}$ F. F. Espinosa, A. H. Shapiro, J. J. Fredberg, and R. D. Kamm, "Spreading of exogenous surfactant in an airway," J. Appl. Physiology 75, 2028 (1993).

${ }^{18}$ M. E. Borgas and J. B. Grotberg, "Monolayer flow on a thin film," J. Fluid Mech. 193, 151 (1988).

${ }^{19}$ M. A. Spaid and G. M. Homsy, "Stability of Newtonian and viscoelastic dynamic contact lines," Phys. Fluids 8, 460 (1996).

${ }^{20}$ D. E. Kataoka and S. M. Troian, "A theoretical study of instabilities at the advancing front of thermally driven coating films," J. Colloid Interface Sci. 192, No. 2, 350 (1997).

${ }^{21}$ W. E. Schiesser, The Numerical Method of Lines (Academic, San Diego, 1991).

${ }^{22}$ D. P. Gaver and J. B. Grotberg, "The dynamics of a localized surfactant on a thin film," J. Fluid Mech. 213, 127 (1990).

${ }^{23}$ V. A. Romanov, "Stability of plane-parallel Couette flow,' Funct. Anal. Appl. 7, 137 (1973).

${ }^{24}$ L. N. Trefethen, A. E. Trefethen, S. C. Reddy, and T. A. Driscoll, "Hydrodynamic stability without eigenvalues," Science 261, 578 (1993). 